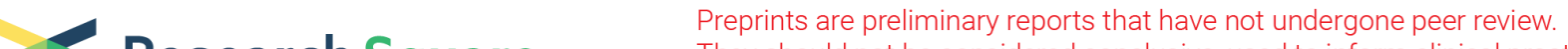 $\begin{array}{ll}\text { Research Square } & \begin{array}{l}\text { They should not be considered conclusive, used to inform clinical practice, } \\ \text { or referenced by the media as validated information. }\end{array}\end{array}$
}

\section{Gene Expression Profiling of Inflammatory Cytokines in Esophageal Biopsies of Different Phenotypes of Gastroesophageal Reflux Disease: a cross-sectional study}

\section{Monica Zavala-Solares}

Instituto Nacional de Ciencias Medicas y Nutricion Salvador Zubiran Departamento de Gastroenterologia

\section{Gabriela Fonseca-Camarillo}

Instituto Nacional de Ciencias Medicas y Nutricion Salvador Zubiran Departamento de Gastroenterologia

\section{Miguel Valdovinos}

Instituto Nacional de Ciencias Medicas y Nutricion Salvador Zubiran Departamento de Gastroenterologia

\section{Julio Granados}

Instituto Nacional de Ciencias Medicas y Nutricion Salvador Zubiran

\section{Guido Grajales-Figueroa}

Instituto Nacional de Ciencias Medicas y Nutricion Salvador Zubiran

\section{Luis Zamora-Nava}

Instituto Nacional de Ciencias Medicas y Nutricion Salvador Zubiran

\section{Nancy Aguilar-Olivos}

Instituto Nacional de Ciencias Medicas y Nutricion Salvador Zubiran

\section{Luis Valdovinos-García}

Instituto Nacional de Ciencias Medicas y Nutricion Salvador Zubiran

Jesus K. Yamamoto-Furusho ( $D$ kazuofurusho@hotmail.com )

Instituto Nacional de Ciencias Medicas y Nutricion Salvador Zubiran https://orcid.org/0000-00025247-5812

\section{Research article}

Keywords: Esophagitis, Non-Erosive Reflux Disease (Nerd), Cytokine, Gene

Posted Date: March 5th, 2020

DOI: https://doi.org/10.21203/rs.3.rs-16050/v1 
License: (c) (i) This work is licensed under a Creative Commons Attribution 4.0 International License. Read Full License

Version of Record: A version of this preprint was published at BMC Gastroenterology on May 3rd, 2021. See the published version at https://doi.org/10.1186/s12876-021-01707-7. 


\section{Abstract}

Background: Patients clinical phenotypes in gastroesophageal reflux disease (GERD) are classified as: Barrett's esophagus (BE), erosive esophagitis (EE) and non-erosive gastroesophageal reflux disease (NERD). The aim of this study was to characterize genes involved in the pathophysiology and immune response of GERD.

Methods: This is an observational and cross-sectional study. All patients with BE, EE, NERD and the control group were subjected to a superior endoscopy (with biopsies of esophageal mucosa). The cytokine mRNA relative quantification of target genes was conducted by RT-PCR. Changes in gene expression were assessed of the genes associated with inflammation in each disease phenotype. Statistical analysis of differential gene expression was performed by using Dunn's Test for Multiple Comparisons. A p value $<0.05$ was considered as significant.

Results: A Total of 98 patients were included and they were divided into the following groups: Group BE 16 (16.33\%), Group EE 23 (23.47\%), Group NERD 42 (42.86\%) and Control Group 17 (17.35\%). When comparing with control group we found: patients with BE showed an increased expression of IL-8 $(P<0.005)$ and higher levels of: IL-1 $\beta, N F-K \beta, I L-10$ and MMP-3, MMP-9 as well; patients with EE had higher levels of IL-1B, IL-6, IL-8 and IL-10 ( $P<0.005)$ and patients with NERD showed a differential gene expression of cytokines Th1, particularly TNF- $\alpha$ and IL-1B $(P<0.005)$ and decreased gene expression of Th2 cytokines such as IL-10, IL-8 and MMP9.

Conclusions: This study demonstrates the differential expression of mediators of inflammation in the esophageal mucosa of patients with the EE and BE phenotype.

\section{Background}

Gastroesophageal reflux disease (GERD) is a multifactorial disease, and it is one of the most frequent pathologies in the outpatient clinic of gastroenterology; it is defined as the presence of heartburn and regurgitation 1 to 2 times per week for at least one month. GERD requires increased esophageal exposure to gastric content. It has been attributed to the fact that its pathophysiology is complex, involving mechanical factors such as the presence of hiatal hernia up to transient relaxations of the lower esophageal sphincter.[1] Patients according to their clinical phenotype are classified as: Barrett's esophagus (BE), erosive esophagitis (EE) and non-erosive gastroesophageal reflux disease (NERD). The severity of GERD injuries and symptoms cannot be predicted solely based on esophageal exposure.[2] Patients display distinctive clinical pictures, some may have erosions whereas others show a non-erosive disease which would suggest that other factors may be involved, therefore It is critical to understand the molecular mechanisms whereby contact with a refluxate impairs esophageal barrier function.[3] Studies performed in a mice model of gastroesophageal reflux showed upregulation of inflammatory-related genes especially those that depend on the NF-KB pathway, with overexpression of subunits (p50 and p65) and NF-KB target genes (matrix metalloproteinases-3 and -9, IL-1 $\beta$, IL-6, and IL-8) confirming activation of 
the NF-KB pathway and downregulation of Cldn1 and Cldn4 in the esophageal epithelium .[3] Recently Bonfiglio et al. [4] provided evidence for 30 independent loci that are involved in molecular pathways with biological relevance to the pathophysiology of GERD. This study proposed initial insights into the genetic background of GERD which was further supported by GWAS analyses that showed that GERD and Barrett's esophagus and Esophageal adenocarcinoma show a substantial overlap in terms of their genetic etiology. Advances in discovery of new pathways involved in the etiopathogenesis of GERD, highlight the crucial role of regulation of local inflammatory responses. The aim of the present study was to characterize critical genes involved in etiopathogenesis and immune response of GERD and to gain insight into the molecular pathways responsible for GERD pathogenesis.

\section{Methods}

\section{Selection of GERD patients}

This is a cross-sectional study of patients with typical symptoms of gastroesophageal reflux disease (heartburn, regurgitation). This research was carried out in the Department of Gastroenterology and endoscopy at Instituto Nacional de Ciencias Médicas y Nutrición Salvador Zubirán (INCMNSZ). All patients who agreed to participate were subjected to a superior endoscopy (already referred by their treating physician for this study) and according to their findings were classified into 3 groups: BE (Prague classification), EE (Classification of Angeles) and NERD. In a subset of patients of the last group was subclassified on abnormal exposure to acid or normal exposure to acid according to a 24-h pH monitoring study.

Selection criteria for patients with GERD include typical symptoms (heartburn and / or regurgitation) at least once a week for not less than 1 year. Subjects were over 18 years of age, both genders and all of them agreed to participate in the study by signing a consent form.

All groups underwent biopsies of esophageal mucosa during the endoscopic procedure. The control group were patients who came with dyspepsia under study, who at the time of their endoscopy presented a macroscopically gastric mucosa without lesions. It was corroborated that its pHmetry was subsequently negative to abnormal exposure to acid reflux.

Endoscopy of the upper gastrointestinal tract (panendoscopy) was performed and classified according to the findings in: BE (Classification of Prague), EE (Classification of Angeles) and non-erosive reflux disease (NERD) (Normal esophageal-gastric junction mucosa). Biopsies of lesions found in the esophagus or biopsy were taken $4-5 \mathrm{~cm}$ from the $Z$ line (in case of normal mucosa). Patients in the NERD group and controls had a 24-h pH monitoring study. If lesions were found, two biopsies were taken: one from the injured region (BE, EE) and another from the adjacent without injure in the same patient (EB, EE) or if no lesions were found $5 \mathrm{~cm}$ above the esophageal mucosal junction of the esophageal mucosa (two biopsies) (NERD and control group). 
A 24-h esophageal pH monitoring study with 1-sensor catheter (GeroFlex, Alpine bioMed, Fountain Valley $\mathrm{CA}, \mathrm{EU}$ ) placed $5 \mathrm{~cm}$ above the lower esophageal sphincter, located by esophageal manometry was performed. Portable recording device (Digitrapper, Medtronic, Parkway, Minneapolis MN, USA). According to the result of the percentage of exposure time at $\mathrm{pH}<4$, they were classified as Abnormal exposure to acid (exposure time percentage $>4.2 \%$ ) or normal exposure to acid (percentage of exposure time $<4.2 \%$ ).

\section{Operational definitions}

BE: patients with long segment $(>3 \mathrm{~cm})$ and short $(<3 \mathrm{~cm})$ segments of the epithelial column located between the upper border of the gastric folds and the proximal part of the $Z$ line and histopathologically confirmed the presence of Intestinal metaplasia in biopsies of the Barrett epithelium segment.

EE: patients with GERD symptoms with erosions or disruptions of the esophageal mucosa of different degrees. (Classification of Angeles)

NERD: GERD symptoms, but no lesions at endoscopy. These patients, in turn, are subclassified in patients with esophageal exposure with normal reflux (not related to acid reflux) and abnormal reflux (presence of abnormal acid reflux or symptomatic association).

Control group (C): Patients without pathology involving their immunity (neoplasms, celiac disease, rheumatic diseases) who present dyspepsia under study, with normal endoscopy (without organic disease) and with normal pHmetry (which excludes gastroesophageal reflux).

\section{Sample Processing and Gene Expression Analysis.}

The esophageal mucosal biopsies taken from endoscopy were immediately placed in RNA later (Ambion, Austin, TX, USA) and stored at $-70^{\circ} \mathrm{C}$ (short-term; $<6$ months) until used. Then total RNA was isolated using High Pure RNA Tissue (Roche Diagnostics, Mannheim, Germany), following the manufacturer's guidelines. Two hundred nanograms of total RNA was reverse transcribed into cDNA with random hexamer primers (Roche Diagnostics, Mannheim, Germany). The methodology employed was based on the previous studies of gene expression [5-7] PCR amplification was carried out with $20 \mathrm{ng}$ of cDNA, 200 nM forward, reverse primer, and Taqman Master Mix (Roche Diagnostics, Mannheim, Germany Roche Diagnostics, Mannheim, Germany) in a final volume of $10 \mu$ (Table 1). PCR reactions were run in a Light Cycler 480 (Roche Diagnostics, Mannheim, Germany) for 45 cycles, each cycle consists in denaturation for 15 seconds at $95^{\circ}$, primer annealing for 15 seconds at $55^{\circ} \mathrm{C}$, and extension for 30 seconds at $72^{\circ} \mathrm{C}$ and cooling 30 seconds at $40^{\circ} \mathrm{C}$.

For q-PCR assays quality control, determination of linearity and reproducibility was evaluated $(\mathrm{VC}<10 \%)$. The mRNA relative quantification of target genes was conducted using the LightCycler software 4.1, according to the 2-delta-delta $\mathrm{Ct}$ method.

Changes in gene expression were assessed and represented by relative gene expression units of target/housekeeping gene in each disease phenotype, inflammatory molecules include TNF-a, IFN-ץ, IL1 $\beta$ 
IL-6, IL-8, IL-10, MMP3, MMP9, NFKB, p50 and p65 subunits

\section{Statistical Analysis}

Gene expression statistical analysis was performed using Dunn's Test of Multiple Comparisons. Results are reported as means \pm SEM. A p value $<0.05$ was considered as significant. Statistical analysis was performed using the program GraphPad Prism 8.0.

Patients with lesions (EE and $\mathrm{BE}$ ) had 2 biopsies: one biopsy of the lesion and another one of healthy mucosa. Expression of each gene was analyzed with Wilcoxon's test, each patient being its own control.

For the gene-for-gene analysis, nonparametric tests will be used, according to the variances for comparison of the differences between the groups.

\section{Results}

\section{Demographic and clinical characteristics.}

Out of 98 patients included, $68 \%$ were women with a median age of 59 years. They were divided into the following groups: Group BE 16 (16.33\%), Group EE 23 (23.47\%), Group NERD 42 (42.86\%) and Control Group 17 (17.35\%). Demographic characteristics by group are detailed in Table 2.

\section{Differential gene expression of pro-inflammatory mediators in patients with GERD.}

Relative gene expression of pro-inflammatory cytokines was detectable and quantifiable by RT-qPCR in biopsies of different phenotypes of gastroesophageal reflux disease and controls.

\section{Gene Expression Profile in Patients with Barrett's Esophagus and controls.}

Patients with BE showed an increased expression of IL-8 compared control group $(P<0.005)$. Also, we detected higher levels of inflammatory mediators such as: IL-1 $\beta$, NF-K $\beta$, IL-10 and MMP-3, MMP-9 in samples of patients with $\mathrm{BE}$ in compared with control group. Figure 1

\section{Gene expression profile in patients with Erosive Esophagitis and Controls.}

Patients with EE had significantly higher levels of mRNA relative gene expression of IL-1B, IL-6, IL-8 and IL-10 compared to controls $(P<0.005)$. Figure 2

\section{Gene Expression Profile in Patients with Non- Erosive reflux Disease and Controls}

Patients with NERD showed a differential gene expression of cytokines Th1, such as TNF-a and IL-1B compared control group $(\mathrm{P}<0.005)$ and decreased gene expression of Th2 Cytokines such as IL-10, IL-8 and MMP9. Figure 3 


\section{Discussion}

In the present study we showed the gene expression of cytokines involved in the pathophysiology and immune response of GERD, patients with BE showed an increased expression of IL-8, IL-1 $\beta$, NF-K $\beta$, IL-10 and MMP-3, patients with EE had higher levels of IL-1B, IL-6, IL-8 and IL-10 and patients with NERD showed a differential gene expression of cytokines Th1, particularly TNF- $a$ and IL-1B and decreased gene expression of Th2 cytokines such as IL-10, IL-8 and MMP9.

Even though, this is a descriptive study, the findings are of interest, as far as we know, additional studies about TH cytokines in the esophageal mucosal inflammatory process.

Although several studies have reported serum levels of inflammatory mediators in patients with GERD.[8], this study analyzed gene expression profiling of inflammatory mediators (IL-1 $\beta$, IL-6, TNF-a, INF- $\gamma$, and NF$\mathrm{K} \beta$ ) associated with damage of esophageal mucosa from patients with 3 different phenotypes of the disease (BE, EE and NERD) and also compared them with those present in a subgroup of patients with a non-erosive variety who were monitored for 24 hours of $\mathrm{pH}$ measures to differentiate cases of esophageal exposure to abnormal acid from normality.

The profile of mediators in BE showed an increased expression of IL-1 $\beta$, IL-8 and IL-10. These results provide understanding of the pathogenesis of Barrett's esophagus and the possible immunoregulatory role of IL-10 associated with the permanent mucosal damage unable to counteract the aggression of other inflammation mediators.

This is in accordance with a previous reported in a mouse model of Barrett's esophagus that showed an increased expression of IL -10, as compared to those in non-Barrett's esophagus while there were no differences in the levels of pro-inflammatory cytokines such as TNF-a or INF-y [9] .

On the other hand, the expression of MMP3 and MMP9 were increased in BE compared to the control group.

The primary function of matrix metalloproteinases (MMP) is in extracellular matrix (ECM) degradation and remodeling. They are secreted by $T$ cells, neutrophils, keratinocytes, monocytes and macrophages. [10]

It has been proposed that MMP-3 6A/5A polymorphism might also been involved in the presentation of patients with $\mathrm{BE} .[11]$

Additionally, MMP-9 could be involved in the pathogenesis of BE and therefore of esophageal adenocarcinoma as well. We found this protease increased although our group of patients with BE did not showed dysplasia. [12] Further studies are required to determine if metalloproteins play a role in mucosal damage in BE. Our study highlights the participation of MMP-9 and MMP-3 in patients with BE, possibly forming part of their pathological mechanism for this change of epithelium. 
In relation to IL-6 measurements, we found an increased expression of this cytokine in patients with BE as compared to controls.

In this context, Isomoto et al [2] highlighted the role of IL-6 as an inflammatory mediator of Th1 response that plays an important role in the malignant progression of the esophageal epithelium. In this sample in the BE phenotype does not highlight this participation probably related to patients not presenting dysplasia but only the change of epithelium corroborated by histology.

Chronic inflammation in BE may play a critical role in the progression from benign to malignant esophageal disease[13]. The rate of progression from Barrett's esophagus to esophageal adenocarcinoma is approximately $0.12-0.4 \%$ per patient-year [14-16].

For this reason, we decided to perform gene expression analysis of nuclear factor NF-k $\beta$ in patients with $\mathrm{BE}$, we found an increased expression of NF-kB in the phenotype of BE compared to the other phenotypes and the control group.

Fang et al [3] proposed an inflammatory profile for esophageal barrier dysfunction by activating NF-kB signaling pathways triggered by mucosal damage from gastroesophageal reflux.

One of the actions of NF-kB is in the proliferation of T cells and in the pro-inflammatory response which in turn acts as a command of the immune response.[17] Definition of the signaling pathways from the release of NF-kB in GERD are still pending.

On the other hand, the erosive phenotype is a classic example of the balance that exists between the expression of Th1 and Th2 response, to the aggression presented (reflux).

In this study we showed an increase expression of pro inflammatory cytokines such as IL-1B, IL-6 and IL8 in mucosal biopsies of patients with EE.

According to our results, Mönkemüller K, et al[18]; showed that IL-1B and IL-8 expression correlate with the histomorphological changes in esophageal mucosa of patients with Erosive and Non-Erosive Reflux Disease.

Previously, Rieder et al[19] demonstrated the increase production of IL-1B and IL-6 in EE compared to controls and Fitzgerald et al[20] reported an inflammatory profile by increased production of IL-1B, IL-8 and INF-Y in esophageal epithelium and neutrophils of EE patients.

Blanchard et al[21] found an increased plasma cytokine levels of IL-8 in patients with EE and healthy subjects.

Interestingly, we found an increased gene expression of IL-10 in mucosa of patients with EE in compared with controls, these results suggest the possible role of IL-10 as a critical cytokine for immunoregulatory mechanism in the inflammatory chronic response in the esophagus. 
Similar to our results, there are reports in Asian populations that show association of IL-1B and IL-10 polymorphisms with an increased risk of erosive reflux esophagitis and gastritis[22]. One of the perspectives of this study is to identify the role of the polymorphisms of IL-1B, IL-8 and IL-10 in Mexican patients with EE and NERD.

Recent studies have provided greater insight into the pathophysiology and symptom generation in NERD[24].

In patients with NERD, we found a significantly increased expression of IL-1B , TNF-a and MMP-9 in compared with control group.

Conversely, we detected a decreased expression of IL-8, IL-10 and MMP-3 in patients with NERD.

Previously, Kanazawa et al[25] in a Japanese study of patients with NERD with minimal mucosal involvement, as determined by endoscopy , IL-8 mRNA levels were increased compared with NERD patients with no mucosal involvement and with control, but in this study is important to note the subgroup with NERD had not been studied by $\mathrm{pH}$ monitoring to truly corroborate this finding in patients who had normal acid exposure.

Interestingly, Kanasawa et al[25] detected an increase expression of IL-8 in NERD compared to asymptomatic subjects without pHmetry studies.[25] Yoshida[26] also found an increase the expression of this chemokine in patients with NERD.

It has also been demonstrated in patients with NERD, that exposure to GER in squamous cells increases the secretion of IL-8 and II-1B causing an increase in the migration of T cells and neutrophils.[23] Previous work in GERD has shown an increase in the expression of IL-1B, TNF- $a$, IL-8 and IL-10. [20, 27, 28]

This study showed the gene expression profiling of inflammatory mediators in the esophagus tissue from patients with different phenotypes of GERD.

Is important to consider transcriptomic profiles and their association with clinical outcomes for the possible application of this technology in the development of personalize medicine.

\section{Conclusions}

This study demonstrated the differential expression of mediators of inflammation in the esophageal mucosa of patients with phenotype EE and BE. As far as the mucosal damage pathways are concerned, this could be mediated by NFKBp50 and NFKBp65 as well as by MMP9, this will lead to mucosal rupture by the family of metalloproteins, and the subfamily of gelatinases. Understanding of this pathway could lead us to the possibility of reverse the alteration of these genes and distinguish them from those that are involved in erosions or lesions from patients with GERD as compared to the NERD phenotype. 


\section{Abbreviations}

BE, Barrett's esophagus; CDNA, complementary DNA; Cldn1, claudin 1; Cldn4 claudin 4; ECM, extracellular matrix; EE, erosive esophagitis; GAPDH, glyceraldehyde-3-phosphate dehydrogenase; GWAS, genomewide association study; IFN-ץ interferon Y ; IL-10 interleukin 10; IL-1 $\beta$, interleukin 1 $\beta$; IL-6 interleukin 6; IL8, interleukin 8; MMP3, matrix metalloproteinase 3; MMP9, matrix metalloproteinase 9; NERD, non-erosive gastroesophageal reflux disease. ; NF-kB , nuclear factor kappa B; RNA , ribonucleic acid; RT-PCR, Real Time Polymerase Chain Reaction; SEM, standard error of the mean ; TNF-a, tumor necrosis factor a

\section{Declarations}

\section{Ethical considerations}

The study was approved by the Ethics Committee of Research of Instituto Nacional de Ciencias Médicas y Nutrición, Salvador Zubirán with reference 987 and a written informed consent was obtained from all patients. This study was performed according to the principles expressed in the Declaration of Helsinki.

\section{Availability of data and materials}

All data and figures used to support the findings of this study are included within the article.

\section{Competing interests}

The authors declare that they have no conflicts of interest with the contents of this article.

\section{Acknowledgements}

We thank Posgrado de Maestría y Doctorado en Ciencias Médicas. Universidad Nacional Autónoma de México and Consejo Nacional de Ciencia y Tecnología (CONACYT). We also thank to Ramón Olavide García, Lol-Be Pinzón Te, Alicia Sofía Villar Chávez for their participation in the pHmeter studies.

\section{Funding:}

This work was supported by funds from the Inflammatory Bowel Disease Clinic at the National Institute of Medical Sciences and Nutrition and grant provided by Consejo Nacional de Ciencia y Tecnología (CONACYT) to Mónica Rocio Zavala Solares.

\section{Author information}

\section{Affiliations}

Programa de Doctorado en Ciencias Médicas, Unidad de Posgrado, Universidad Nacional Autónoma de México, México City, México. 
Department of Gastroenterology, Department of Transplantation and Department of Endoscopy. Instituto Nacional de Ciencias Médicas y Nutrición, Salvador Zubirán, México City, México

\section{Authors' contributions:}

MRZS, Conceptualization, Data curation, Formal analysis, Investigation, Methodology, Project administration, Writing - original draft, Writing - review \& editing; GFC, Performed the PCR-RT experiments of gene expression analysis, Data curation, Formal analysis, Investigation, Writing - original draft, Writing - review \& editing;MAVD, Conceptualization, Supervision, Writing - review \& editing, GGF, Data curation, Investigation, Writing - review \& editing; LEZN Data curation, Investigation, Writing review \& editing; NEAO, Data curation, Investigation, Writing - review \& editing; LRVG, Data curation, Investigation, Writing - review \& editing, JG, Supervision, Visualization, Writing - review \& editing; JKYF Conceptualization, Data curation, Formal analysis, Funding acquisition, Resources, Investigation, Methodology, Project administration, Supervision, Validation, Writing - original draft, Writing - review \& editing

All authors have read and approved the manuscript.

\section{References}

1. Tack J, Pandolfino JE. Pathophysiology of Gastroesophageal Reflux Disease. Gastroenterology 2018,154:277-288.

2. Isomoto H, Nishi Y, Kanazawa Y, Shikuwa S, Mizuta Y, Inoue K, et al. Immune and Inflammatory Responses in GERD and Lansoprazole. J Clin Biochem Nutr 2007,41:84-91.

3. Fang Y, Chen H, Hu Y, Djukic Z, Tevebaugh W, Shaheen NJ, et al. Gastroesophageal reflux activates the NF-kappaB pathway and impairs esophageal barrier function in mice. Am J Physiol Gastrointest Liver Physiol 2013,305:G58-65.

4. Bohmer AC, Schumacher J. Insights into the genetics of gastroesophageal reflux disease (GERD) and GERD-related disorders. Neurogastroenterol Motil 2017,29.

5. Fonseca-Camarillo G, Furuzawa-Carballeda J, Iturriaga-Goyon E, Yamamoto-Furusho JK. Differential Expression of IL-36 Family Members and IL-38 by Immune and Nonimmune Cells in Patients with Active Inflammatory Bowel Disease. Biomed Res Int 2018,2018:5140691.

6. Fonseca-Camarillo G, Furuzawa-Carballeda J, Yamamoto-Furusho JK. Interleukin 35 (IL-35) and IL37: Intestinal and peripheral expression by $\mathrm{T}$ and $\mathrm{B}$ regulatory cells in patients with Inflammatory Bowel Disease. Cytokine 2015,75:389-402.

7. Yamamoto-Furusho JK, Fonseca-Camarillo G, Furuzawa-Carballeda J, Sarmiento-Aguilar A, BarretoZuniga R, Martinez-Benitez B, et al. Caspase recruitment domain (CARD) family (CARD9, CARD10, CARD11, CARD14 and CARD15) are increased during active inflammation in patients with inflammatory bowel disease. J Inflamm (Lond) 2018,15:13. 
8. Deng Y, Pan L, Qian W. Associations between the severity of reflux esophagitis in children and changes in oxidative stress, serum inflammation, vasoactive intestinal peptide and motilin. Exp Ther Med 2019,18:3509-3513.

9. Kohata Y, Fujiwara Y, Machida H, Okazaki H, Yamagami H, Tanigawa T, et al. Role of Th-2 cytokines in the development of Barrett's esophagus in rats. J Gastroentero/ 2011,46:883-893.

10. Sengupta N, MacDonald TT. The role of matrix metalloproteinases in stromal/epithelial interactions in the gut. Physiology (Bethesda) 2007,22:401-409.

11. Cheung WY, Zhai R, Bradbury P, Hopkins J, Kulke MH, Heist RS, et al. Single nucleotide polymorphisms in the matrix metalloproteinase gene family and the frequency and duration of gastroesophageal reflux disease influence the risk of esophageal adenocarcinoma. Int $J$ Cancer 2012,131:2478-2486.

12. Davelaar AL, Straub D, Buttar NS, Fockens P, Krishnadath KK. Active matrix metalloproteases are expressed early on and are high during the Barrett's esophagus malignancy sequence. Scand $J$ Gastroenterol 2015,50:321-332.

13. Yang L, Francois F, Pei Z. Molecular pathways: pathogenesis and clinical implications of microbiome alteration in esophagitis and Barrett esophagus. Clin Cancer Res 2012,18:2138-2144.

14. O'Connor JB, Falk GW, Richter JE. The incidence of adenocarcinoma and dysplasia in Barrett's esophagus: report on the Cleveland Clinic Barrett's Esophagus Registry. Am J Gastroenterol 1999,94:2037-2042.

15. Drewitz DJ, Sampliner RE, Garewal HS. The incidence of adenocarcinoma in Barrett's esophagus: a prospective study of 170 patients followed 4.8 years. Am J Gastroenterol 1997,92:212-215.

16. Hvid-Jensen F, Pedersen L, Drewes AM, Sorensen HT, Funch-Jensen P. Incidence of adenocarcinoma among patients with Barrett's esophagus. N Engl J Med 2011,365:1375-1383.

17. D'Ignazio L, Bandarra D, Rocha S. NF-kappaB and HIF crosstalk in immune responses. FEBS J 2016,283:413-424.

18. Monkemuller K, Wex T, Kuester D, Fry LC, Peitz U, Beyer M, et al. Interleukin-1beta and interleukin-8 expression correlate with the histomorphological changes in esophageal mucosa of patients with erosive and non-erosive reflux disease. Digestion 2009,79:186-195.

19. Rieder F, Cheng L, Harnett KM, Chak A, Cooper GS, Isenberg G, et al. Gastroesophageal reflux diseaseassociated esophagitis induces endogenous cytokine production leading to motor abnormalities. Gastroenterology 2007,132:154-165.

20. Fitzgerald RC, Onwuegbusi BA, Bajaj-Elliott M, Saeed IT, Burnham WR, Farthing MJ. Diversity in the oesophageal phenotypic response to gastro-oesophageal reflux: immunological determinants. Gut 2002,50:451-459.

21. Blanchard C, Stucke EM, Rodriguez-Jimenez B, Burwinkel K, Collins MH, Ahrens A, et al. A striking local esophageal cytokine expression profile in eosinophilic esophagitis. J Allergy Clin Immunol 2011,127:208-217, 217 e201-207. 
22. Cheng HH, Chang CS, Wang HJ, Wang WC. Interleukin-1 beta and -10 polymorphisms influence erosive reflux esophagitis and gastritis in Taiwanese patients. J Gastroenterol Hepatol 2010,25:14431451.

23. Souza RF, Huo X, Mittal V, Schuler CM, Carmack SW, Zhang HY, et al. Gastroesophageal reflux might cause esophagitis through a cytokine-mediated mechanism rather than caustic acid injury. Gastroenterology 2009,137:1776-1784.

24. Chen CL, Hsu PI. Current advances in the diagnosis and treatment of nonerosive reflux disease. Gastroenterol Res Pract 2013,2013:653989.

25. Kanazawa Y, Isomoto H, Wen CY, Wang AP, Saenko VA, Ohtsuru A, et al. Impact of endoscopically minimal involvement on IL-8 mRNA expression in esophageal mucosa of patients with non-erosive reflux disease. World J Gastroenterol 2003,9:2801-2804.

26. Yoshida N. Inflammation and oxidative stress in gastroesophageal reflux disease. J Clin Biochem Nutr 2007,40:13-23.

27. Rieder F, Biancani P, Harnett K, Yerian L, Falk GW. Inflammatory mediators in gastroesophageal reflux disease: impact on esophageal motility, fibrosis, and carcinogenesis. Am J Physiol Gastrointest Liver Physiol 2010,298:G571-581.

28. Isomoto H, Saenko VA, Kanazawa Y, Nishi Y, Ohtsuru A, Inoue K, et al. Enhanced expression of interleukin-8 and activation of nuclear factor kappa-B in endoscopy-negative gastroesophageal reflux disease. Am J Gastroentero/ 2004,99:589-597.

\section{Tables}

Due to technical limitations, all table files are available for download from the Supplementary File section.

\section{Figures}


$1.60 \mathrm{E}-01$

Gene Expression Profile in Patients with Barrett Esophagus (BE) and controls.

政

1.40E-01

1.20E-01

1.00E-01

8.00E-02

$6.00 \mathrm{E}-02$

4.00E-02

2.00E-02

$0.00 \mathrm{E}+00$

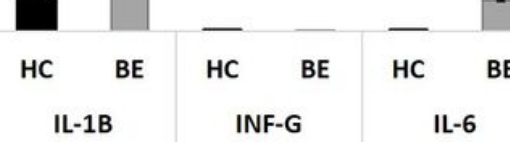
IL-6

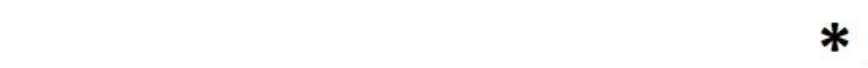

*
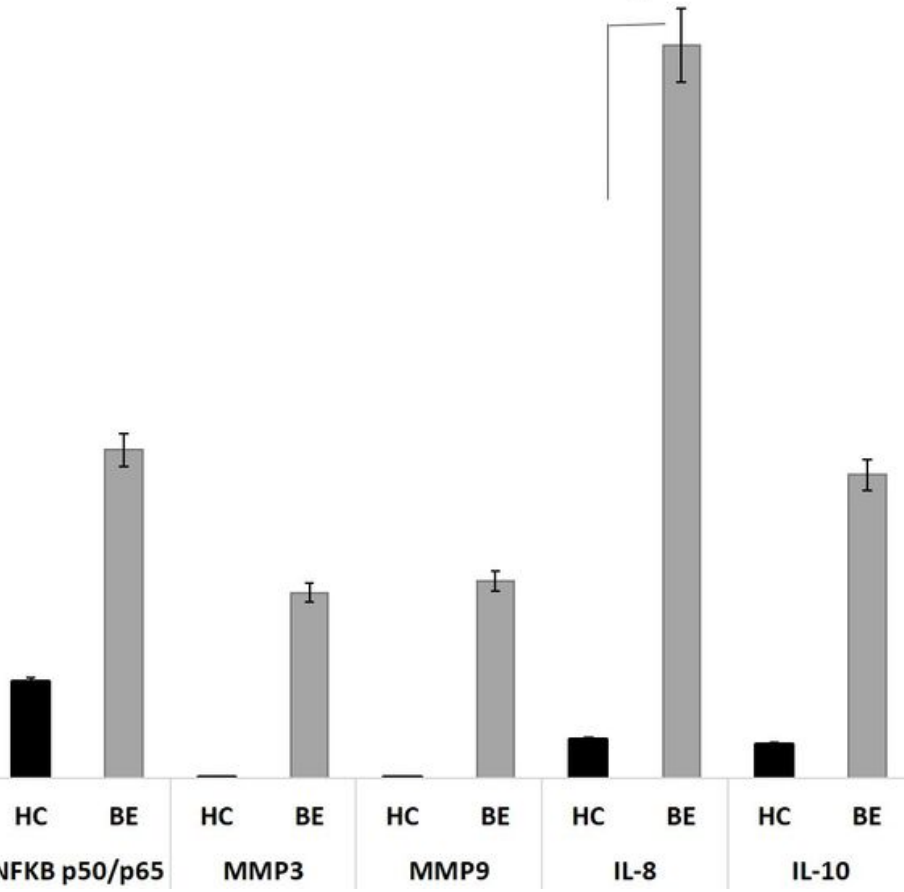

Figure 1

Gene expression profile with Barrett's esophagus (BE) and healthy controls (HC) mRNA levels. Bars show mean \pm SEM of the mean of transcript levels in colonic mucosa from BE patients with GAPDH as housekeeping gene determined by $2-\Delta \Delta \mathrm{Ct} * \mathrm{p}<0.05$

2.50E-02

Gene Expression Profile in Patients with Erosive Esophagitis (EE) and controls

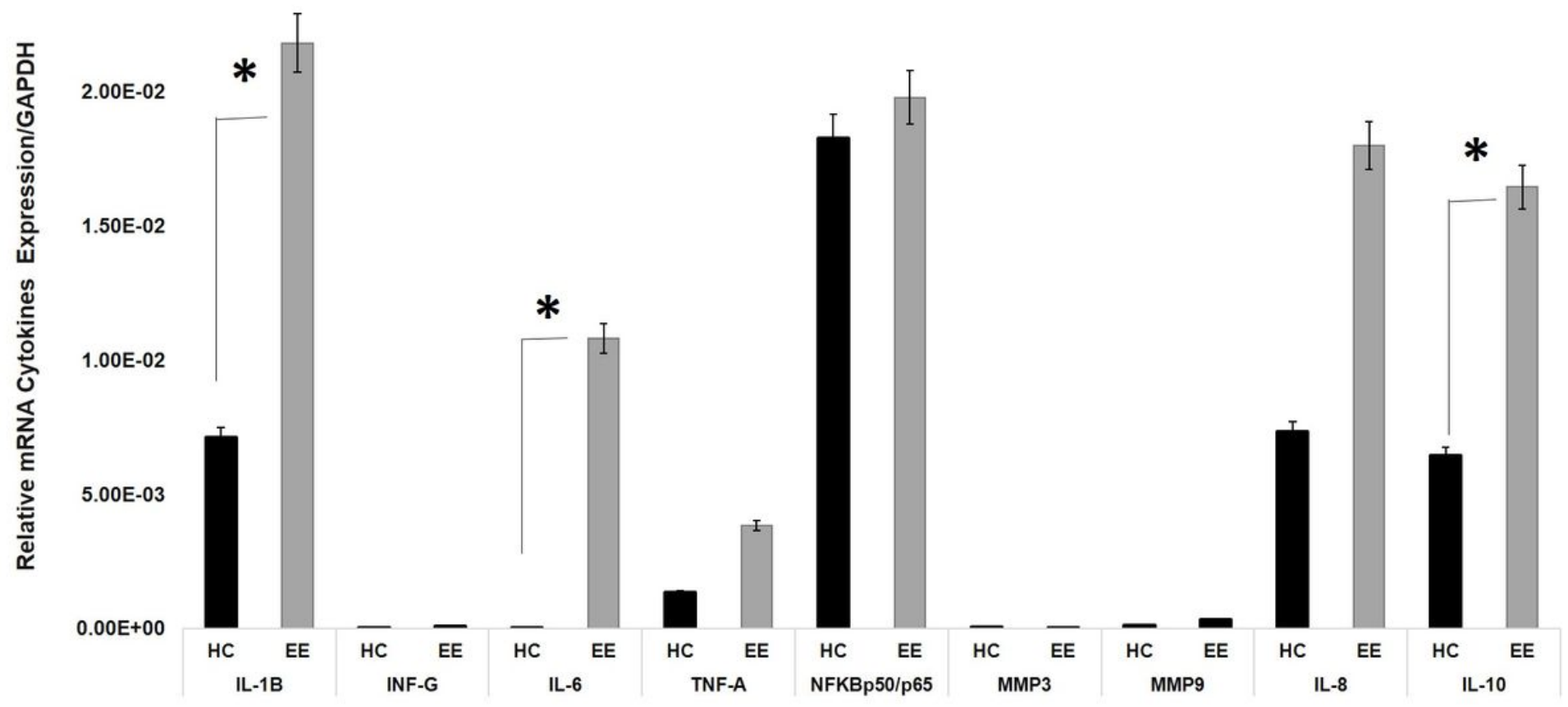


Figure 2

Gene expression profile with Erosive esophagitis (EE) and controls (HC) mRNA levels. Bars show mean \pm SEM of the mean of transcript levels in colonic mucosa from BE patients with GAPDH as housekeeping gene determined by $2-\Delta \Delta \mathrm{Ct} * \mathrm{p}<0.05$

Gene Expression Profile in Patients with Non- Erosive reflux Disease (NERD) and controls.

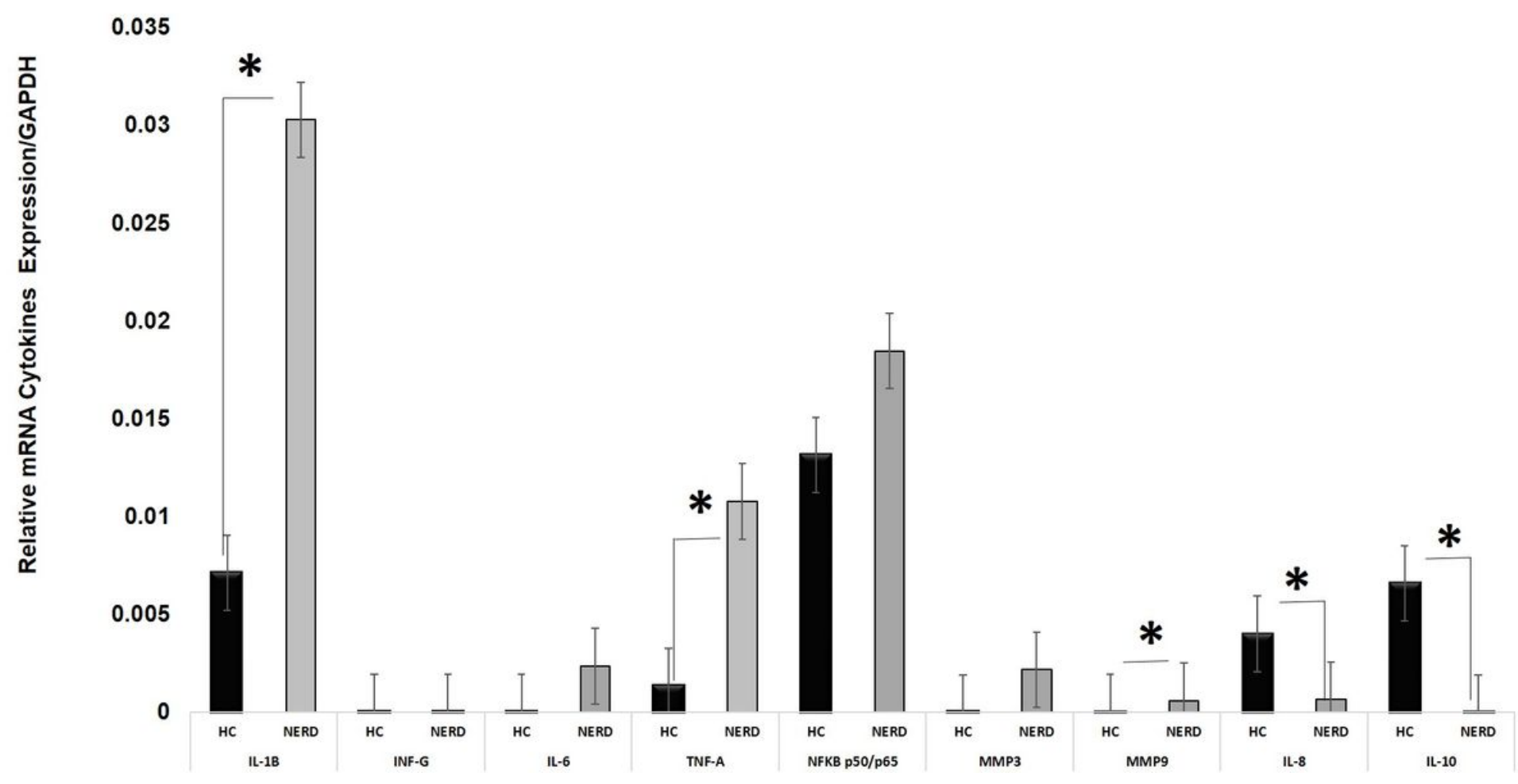

Figure 3

Gene expression profile with Non-erosive reflux disease (NERD) and healthy controls (HC) mRNA levels. Bars show mean \pm SEM of the mean of transcript levels in colonic mucosa from BE patients with GAPDH as housekeeping gene determined by $2-\Delta \Delta \mathrm{Ct} * \mathrm{p}<0.05$

\section{Supplementary Files}

This is a list of supplementary files associated with this preprint. Click to download.

- TABLES.docx

- STROBEchecklistcrosssectional1.docx 